\title{
Diffusion at constant speed in a model phase space
}

\author{
S. Anantha Ramakrishnat and N. Kumar \\ Raman Research Institute, C.V. Raman Avenue, Bangalore 560 080, India
}

(November 21, 2018)

\begin{abstract}
We reconsider the problem of diffusion of particles at constant speed and present a generalization of the Telegrapher process to higher dimensional stochastic media $(d>1)$, where the particle can move along $2^{d}$ directions. We derive the equations for the probability density function using the "formulae of differentiation" of Shapiro and Loginov. The model is an advancement over similiar models of photon migration in multiply scattering media in that it results in a true diffusion at constant speed in the limit of large dimensions.
\end{abstract}

\section{INTRODUCTION}

Diffusion theory is an old subject and has been applied with enormous success in many fields of Physics, Chemistry and Biology. But pure diffusion of particles is actually unphysical as it neglects the inertia of the particles. It is a Wiener process for the spatial position of the particles and holds strictly in the limit when the trasnport mean free path $l^{*} \rightarrow 0$ and the speed $c \rightarrow \infty$ such that the diffusion coefficient $D=c l^{*} / 3$ is a constant. In other words the particle scatters at every point and has no directional memory or persistence. The persistence becomes important in many problems when processes at short length and time scales become important as in polymer physics and radiative transport in random media.

*Presently at the Blackett Laboratory, Imperial College, London SW7 2BZ, U.K. 
The incoherent energy transport of light in random media described by the radiative transfer equation [1.2] can be approximated as a diffusion process in a coarse grained description. But again, the diffusion approximation fails here at short length- and time-scales [3], not because of any finite photon mass, but due to the anisotropic (forward) scattering nature of the finite size scatterers. This becomes particularly important in the biomedical imaging and diagnostic applications with NIR light [4] when the ballistic and 'snake' (near ballistic) light in highly forward scattering media are used. Since the general analytic solutions to the radiative transfer equation that take into account the full scattering properties are not known even for simple geometries, it becomes important to develop simple models that incorporate the physical persistence of the problem into the stochastic process. Further in the case of light, assuming the distance between the scatterers is much larger than the wavelength of the light, the speed of the photon is constant in between the scattering events. While approximately describing light as a particle undergoing stochastic motion, this constancy of the speed should be preserved at the very least.

Recently we successfully described this diffusion at a constant speed of photons as a diffusion on the velocity sphere [5]. Among the other models proposed to deal with the intermediate range of length- and time-scales between the ballistic motion and diffusive transport, the attempts to generalize the Telegrapher equation are important. The Telegrapher equation is exact in one dimension and describes the diffusion of a particle whose speed is fixed, i.e., the velocity can take on only two values $\pm c$ [6]. The Telegrapher equation, i.e,

$$
\frac{\partial^{2} P}{\partial t^{2}}+\Gamma \frac{\partial P}{\partial t}-c^{2} \frac{\partial^{2} P}{\partial x^{2}}=0
$$

where $\mathrm{P}$ is the probability distribution function and $\Gamma / 2$ is the mean scattering rate, is a combination of the wave equation describing the inertial (persistent) aspect and of the diffusion equation describing the stochastic aspect. This equation has found wide application in many fields [7] and was first considered by J.C. Maxwell [8.9], more than a century ago in his attempt to describe heat conduction from basic kinetic theory. He discarded the inertial term, however, on the grounds that it would be important only at extremely short time 
scales. It also has since been shown to describe the 'second sound' in liquid Helium II, and classical mesoscopic diffusion obeying the Maxwell-Cattaneo law instead of the usual Fick's law 10$]$.

Following a suggestion of Ishimaru [11], a generalization of the Telegrapher equation to higher dimensions in a heuristic manner was attempted [12] by simply replacing $\partial^{2} P / \partial x^{2}$ in Eqn.(7.1) by $\nabla^{2} P$, to describe photon migration at short length scales, by including some ballistic aspects. This ad-hoc generalization appeared to be quite successful to describing photon migration as it preserved causality and did much better than the diffusion approximation to describe photon transport in absorbing media [12,13. This was also extended to studies of Diffusing Wave Spectroscopy in thin samples [14. It was, however, shown by comparing it with Monte-Carlo simulations that this generalization furnished no better an approximation than the diffusion approximation in higher dimensions [15]. The in-principle weakness of this 'ad-hoc' approach was demonstrated by the fact that the photon probability density evolving under this equation becomes negative in two dimensions for the simplest case of an unbounded (infinite) medium at short times $\left(t \sim t^{*}\right)$ when the ballistic aspects of transport are most important. In fact, the negativity of the solution to this equation is a generic property in even-dimensional spaces [15,16].

In this paper, we reconsider the problem of diffusion of photons at constant speed and present a generalization of the Telegrapher process to higher dimensional turbid media $(d>$ 1 ), where the photon can move along $2^{d}$ directions along the diagonals of a $d$-dimensional hypercube. We derive the equation for the probability density function using the "formulae of differentiation" of Shapiro and Loginov [17], by considering a correlated random walk at constant speed. We show that a partial differential equation of order $2^{d}$ results for the probability distribution function in $d$-dimensions. Our model is an advancement over the earlier models of Boguñá et al. [18,19, where the photon could only move along the $2 d$ directions along the axes, and results in a true diffusion at constant speed in the limit of large dimensions. Our work brings out certain features that were not recognized in earlier work. Light in the stochastic medium is considered to be a particle on which the medium 
exerts fluctuating forces. Each scattering event only changes the direction of the photon without affecting the speed of propagation.

\section{THE TELEGRAPHER PROCESS IN ONE DIMENSION}

Let us first consider the dynamics of a particle executing a random walk in one dimension while moving with constant speed $c$. This would describe the motion of light in a disordered fibre, or of electrons on the Fermi points in a one-dimensional disordered wire, if we neglect the wave nature and the consequent Anderson localization (Strictly speaking, this description would not hold for 1-D where all the quantum states are localized states. For sample lengths much smaller than the localization length, however, the transport is almost diffusive). The velocity $v(t)$ of the particle is a random function of time such that it can take only two values $\pm c$, i.e., a Dichotomic Markov process. If $\Gamma / 2$ be the transition probability per unit time between these two values of the velocity $(\langle\rangle$ indicate averaging over the disorder),

$$
\begin{aligned}
\langle v(t)\rangle & =0, \\
\left\langle v(t) v\left(t^{\prime}\right)\right\rangle & =c^{2} \exp \left(-\Gamma\left|t-t^{\prime}\right|\right),
\end{aligned}
$$

i.e. the velocity is exponentially correlated in time. We note that the stochastic Langevin equation for the displacement $\dot{x}=v(t)$ gives:

$$
\begin{aligned}
\langle x\rangle & =0 \\
\left\langle x^{2}\right\rangle & =\frac{2 c^{2}}{\Gamma}\left(t-\frac{1-e^{-\Gamma t}}{\Gamma}\right),
\end{aligned}
$$

i.e., the behaviour at long times $(t \rightarrow \infty)$ or very large scattering strengths (large $\Gamma$ ) is diffusive $\left(\left\langle x^{2}\right\rangle \sim t\right)$ and at short times $(t \rightarrow 0)$, the behaviour is ballistic $\left(\left\langle x^{2}\right\rangle \sim t^{2}\right)$.

Next we will derive the equation for the probability distribution function. Let $\Pi(x ; t)$ be the phase space density of points in the $\mathrm{x}$-t phase space. Now, $\Pi$ satisfies the Stochastic Liouville equation :

$$
\frac{\partial \Pi}{\partial t}+\frac{\partial}{\partial x}(\dot{x} \Pi)=0
$$


Averaging over all the realizations of the random function $v(t)$, by the van Kampen lemma [20], the probability distribution $P(x ; t)=\langle\Pi(x ; t)\rangle$. We also define $W(x ; t)=\langle v(t) \Pi(x ; t)\rangle$ and obtain,

$$
\frac{\partial P}{\partial t}+\frac{\partial W}{\partial x}=0
$$

Now using the "formulae of differentiation" of Shapiro and Loginov [17], for a Dichotomic Markov process,

$$
\frac{\partial W}{\partial t}=-\Gamma W-c^{2} \frac{\partial P}{\partial x}
$$

Eliminating $\mathrm{W}$ from the above equations, we obtain

$$
\frac{\partial^{2} P}{\partial t^{2}}+\Gamma \frac{\partial P}{\partial t}=c^{2} \frac{\partial^{2} P}{\partial x^{2}}
$$

i.e. the Telegrapher equation for the probability distribution function $P$. This is an exact description of the motion of a particle with constant speed in 1-D.

The solutions to Equation.(9) are well known and, in an infinite medium given by [6, 12

$$
P(x, t ; x=0, t=0)=\left(\frac{\Gamma}{2 c}\right) e^{-\Gamma t / 2}\left[I_{0}(y)+2 \Gamma t \frac{I_{1}(y)}{y}\right] \theta(c t-|x|),
$$

where $y=(\Gamma / 2 c) \sqrt{c^{2} t^{2}-x^{2}}, I_{0}$ and $I_{1}$ are the modified Bessel functions of order zero and one respectively, and $\theta$ is the Heaviside step function. Note that the solution is zero for $|x|>c t$ and thus causality is preserved. The solution indicates that the particles spread out symmetrically from the origin, half of them to the left and the other half to the right, with a front velocity of $c$ beyond which there are no more particles and heap up at the 'light fronts' where there are $N / 2 \exp (-\Gamma t / 2)$ particles (if there are $N$ particles altogether).

\section{GENERALIZATION TO HIGHER DIMENSIONS}

Now, we seek a generalization of the Telegrapher process process to higher dimensions. The simplest way of doing this is to make every orthogonal component a dichotomic Markov process which take the values $\pm c$. Thus, in $d$ dimensions, the particle is seen to move along 
the diagonals of the $d$ dimensional hypercube with a speed $\sqrt{d} c$. Here the real space is continuous, while the velocity space is discrete and can take only $2^{d}$ discrete values in $d$ dimensions. This is the model phase space that we consider the photon to execute a random walk in. For example, in Fig. 1, we show a possible trajectory in the real space for $d=2$ and a particle released from the origin at $t=0$. 


\section{FIGURES}

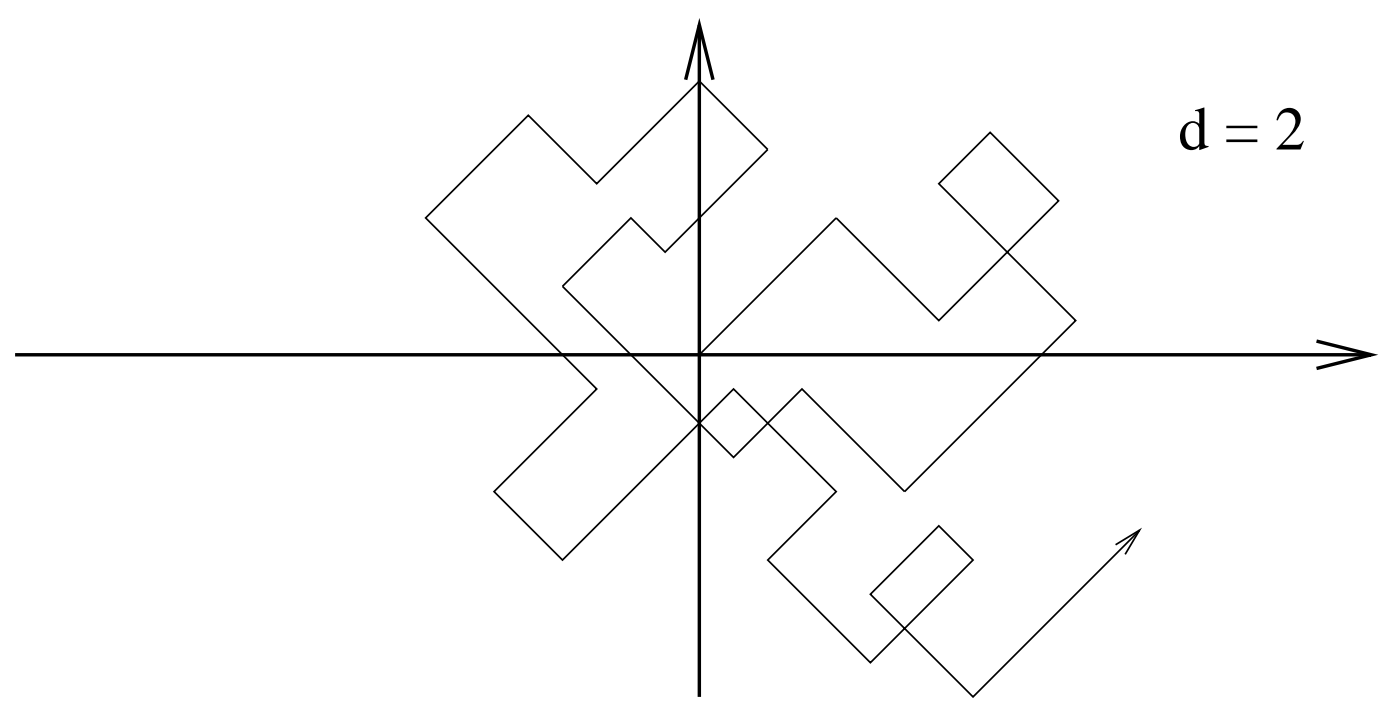

FIG. 1. One realization of the possible real space trajectories for the generalized Telegrapher process in two dimensions. 


\section{A. The equations for the generalized Telegrapher process}

In two dimensions, we will consider both the $\mathrm{x}$ and $\mathrm{y}$ components of the velocity of the particle to be independent dichotomic Markov processes,

$$
\begin{aligned}
v_{x}(t) & =c \chi_{1}(t), \\
v_{y}(T) & =c \chi_{2}(t), \\
\left\langle\chi_{i}(t)\right\rangle & =0, \\
\left\langle\chi_{i}(t) \chi_{j}\left(t^{\prime}\right)\right\rangle & =\delta_{i j} \exp \left(-\Gamma\left|t-t^{\prime}\right|\right),
\end{aligned}
$$

where $\chi_{i}(t)$ are unimodular processes. The particle is thus seen to move along the four directions $( \pm \hat{i}, \pm \hat{j})$ with a constant speed $\sqrt{2} c$. Now using the stochastic Louiville equation, We define the averages $P(x, y ; t)=\langle\Pi(x, y ; t)\rangle, W_{x}=\left\langle v_{x}(t) \Pi(x, y ; t)\right\rangle, W_{y}=\left\langle v_{y}(t) \Pi(x, y ; t)\right\rangle$

and $W_{x y}=\left\langle v_{x}(t) v_{y}(t) \Pi(x, y ; t)\right\rangle$. We generalize the "formula of differentiation" of Shapiro and Loginov to n-independent dichotomic Markov processes as

$$
\begin{aligned}
\frac{d}{d t} & \left\langle v_{1} v_{2} \cdots v_{n} \Pi\left[v_{1}, v_{2}, \cdots v_{n}\right]\right\rangle_{v_{1}, v_{2}, \cdots, v_{n}}= \\
& =-\left(\Gamma_{1}+\Gamma_{2}+\cdots+\Gamma_{n}\right)\left\langle v_{1} v_{2} \cdots v_{n} \Pi\left[v_{1}, v_{2}, \cdots, v_{n}\right]\right\rangle_{v_{1}, v_{2}, \cdots v_{n}} \\
& +\left\langle v_{1} v_{2} \cdots v_{n} \frac{d \Pi\left[v_{1}, v_{2}, \cdots v_{n}\right]}{d t}\right\rangle_{v_{1}, v_{2}, \cdots, v_{n}}
\end{aligned}
$$

where $v_{i}(t)$ are independent dichotomic Markov processes, $\Gamma_{i}$ are the respective transition rates and $\Pi$ is a functional of $v_{1}, v_{2}, \cdots, v_{n}$. Using the above, we obtain the following closed set of equations :

$$
\begin{aligned}
\frac{\partial P}{\partial t}+\frac{\partial W_{x}}{\partial x}+\frac{\partial W_{y}}{\partial y} & =0, \\
\frac{\partial W_{x}}{\partial t}+\Gamma W_{x} & =-c^{2} \frac{\partial P}{\partial x}-\frac{\partial W_{x y}}{\partial y}, \\
\frac{\partial W_{y}}{\partial t}+\Gamma W_{y} & =-c^{2} \frac{\partial P}{\partial y}-\frac{\partial W_{x y}}{\partial x}, \\
\frac{\partial W_{x y}}{\partial t}+2 \Gamma W_{x y} & =-c^{2}\left[\frac{\partial W_{y}}{\partial x}+\frac{\partial W_{x}}{\partial y}\right] .
\end{aligned}
$$

Eliminating $W_{x}, W_{y}, W_{x y}$ from the above set of equations, we obtain for the probability distribution function $P(x, y ; t)$ : 


$$
\frac{\partial}{\partial t}\left(\frac{\partial}{\partial t}+2 \Gamma\right)\left(\frac{\partial}{\partial t}+\Gamma\right)^{2} P-2 c^{2}\left(\frac{\partial}{\partial t}+\Gamma\right)^{2} \nabla^{2} P+c^{4}\left(\frac{\partial^{2}}{\partial x^{2}}-\frac{\partial^{2}}{\partial y^{2}}\right) P=0
$$

By performing a $\pi / 4$ rotation of the space axes and rescaling the speed to $c$, this equation is seen to be the same as the one derived by Bog̃una et al. [18] by a different approach. Unlike the "ad-hoc Generalized Telegrapher equation" of Durian and Rudnick [12], this partial differential equation is of fourth order involving all space and time derivatives.

Similiarly, in three dimensions, we consider the $\mathrm{x}, \mathrm{y}$ and $\mathrm{z}$ components to the velocity to be independent dichotomic Markov processes. Again, following the above procedure, we obtain the closed set of eight equations :

$$
\begin{aligned}
\frac{\partial P}{\partial t}+\frac{\partial W_{x}}{\partial x}+\frac{\partial W_{y}}{\partial y}+\frac{\partial W_{z}}{\partial z} & =0 \\
\frac{\partial W_{x}}{\partial t}+\Gamma W_{x} & =-c^{2} \frac{\partial P}{\partial x}-\frac{\partial W_{x y}}{\partial y}-\frac{\partial W_{z x}}{\partial z} \\
\frac{\partial W_{y}}{\partial t}+\Gamma W_{y} & =-c^{2} \frac{\partial P}{\partial y}-\frac{\partial W_{x y}}{\partial x}-\frac{\partial W_{y z}}{\partial z} \\
\frac{\partial W_{z}}{\partial t}+\Gamma W_{y} & =-c^{2} \frac{\partial P}{\partial z}-\frac{\partial W_{z x}}{\partial x}-\frac{\partial W_{y z}}{\partial y} \\
\frac{\partial W_{x y}}{\partial t}+2 \Gamma W_{x y} & =-c^{2}\left[\frac{\partial W_{y}}{\partial x}+\frac{\partial W_{x}}{\partial y}\right]-\frac{\partial W_{x y z}}{\partial z} \\
\frac{\partial W_{y z}}{\partial t}+2 \Gamma W_{y z} & =-c^{2}\left[\frac{\partial W_{y}}{\partial z}+\frac{\partial W_{z}}{\partial y}\right]-\frac{\partial W_{x y z}}{\partial x} \\
\frac{\partial W_{z x}}{\partial t}+2 \Gamma W_{z x} & =-c^{2}\left[\frac{\partial W_{z}}{\partial x}+\frac{\partial W_{x}}{\partial z}\right]-\frac{\partial W_{x y z}}{\partial y} \\
\frac{\partial W_{x y z}}{\partial t}+3 \Gamma W_{x y z} & =-c^{2}\left[\frac{\partial W_{y z}}{\partial x}+\frac{\partial W_{z x}}{\partial y}+\frac{\partial W_{x y}}{\partial z}\right]
\end{aligned}
$$

It is possible to obtain a cumbersome-looking partial differential equation for $\mathrm{P}(\mathrm{x}, \mathrm{y}, \mathrm{z} ; \mathrm{t})$ alone, similiar to Equation(20) by eliminating the other functions from the above coupled set of differential equations. However, no extra information results and it will not be presented here.

The partial differential equation for $P(\vec{r} ; t)$ alone, in general, will be of order $2^{d}$ (there are $2^{d}$ independent first order coupled differential equations for $d$ dimensions). The set of coupled first order differential equations(21 28) offer a very convenient factorization of the $2^{d}$ dimensional equation satisfied by $P(\vec{r} ; t)$ and are a more convenient starting point for 
numerical calculations of the solutions. We note that the above equations are linear with constant coefficients and can easily be solved by taking Laplace transforms with respect to time and space variables. Inverting the solutions obtained back to the real space-time, however, is non-trivial and only a few characteristic quantities such as the moments of the residence times have been calculated [19] for a similiar model.

\section{B. Absorbing boundary conditions}

For the above system of equations, absorbing boundary conditions can be easily and rigorously applied for this set of equations. For an absorbing boundary at $x=0$, with the stochastic medium occupying the negative semi-infinite half-space, the appropriate boundary conditions corresponding to no incoming flux are

$$
\begin{array}{r}
W_{x}(x=0, y, z ; t)=c P(x=0, y, z ; t), \\
W_{x y}(x=0, y, z ; t)=c W_{y}(x=0, y, z ; t), \\
W_{z x}(x=0, y, z ; t)=W_{z}(x=0, y, z ; t), \\
W_{x y z}(x=0, y, z ; t)=c W_{y z}(x=0, y, z ; t),
\end{array}
$$

and free boundary conditions on the other functions $P, W_{y}, W_{z}, W_{y z}$. These conditions are equivalent to the integral boundary condition $\partial / \partial t \int_{-\infty}^{x=0} P(x, y, z ; t) d x=c P(x=0, y, z ; t)$ on $P(x, y, z ; t)$ alone.

\section{Projected motion along any axis and angular non-symmetry of the model}

In higher dimensions $(d>1)$, the ad-hoc generalized Telegrapher equation viz. $\frac{\partial^{2} P}{\partial t^{2}}+$ $\Gamma \frac{\partial P}{\partial t}-c^{2} \nabla^{2} P=0$ is indeed obtained only if higher order velocity correlations are neglected, i.e., terms such as $W_{x y}=\left\langle v_{x} \Pi\right\rangle, W_{x y z}$ etc. are set to zero. This is not correct especially at short times, when we expect the velocity components to be correlated to a quite some extent. 
However, it is easily seen that the marginal probability distribution for the projected motion along one of the axis $p\left(x_{1} ; t\right)=\int P\left(x_{1}, x_{2}, \cdots, x_{d}\right) d x_{1} d x_{2} \cdots d x_{d}$ satisfies the Telegrapher equation $\frac{\partial^{2} p}{\partial t^{2}}+\Gamma \frac{\partial p}{\partial t}-c^{2} \frac{\partial^{2} p}{\partial x^{2}}=0$. The partial differential equation for $P(\vec{r} ; t)$ alone, in general, will be of order $2^{d}$ (there are $2^{d}$ independent first order coupled differential equations for $d$ dimensions) corresponding to $2^{d}$ directions.

There are some subtle differences between our model and that of Boguná et al [18]. First of all, the number of allowed directions for the photon motion is greater in our model $\left(2^{d}\right)$ than theirs $(2 d)$. The reason is that, they consider that the motion of the particle to be along the axes, while in our case, the motion is along the diagonals of the $d$ dimensional hypercube. They do not obtain a Telegrapher equation for the marginal probability distribution for the projected motion in general as we do. We always have a Telegrapher process along any one axis. This can be best compared in two dimensions by carrying out a $\pi / 4$ rotation of the axes in Eqns.(16-19) and then looking at the projected motion along the axes. We obtain the Telegrapher equation $\frac{\partial^{2} p}{\partial t^{2}}+2 \Gamma \frac{\partial p}{\partial t}-c^{2} \frac{\partial^{2} p}{\partial x^{2}}=0$, i.e., only the diffusion coefficient is renormalized. This corresponds to the three step Telegrapher process of moving at constant speed to the left or the right with a probability $1 / 4$ and being at rest with a probability $1 / 2$. In higher dimensions $(d>2)$, the diagonals of the hypercube are not orthogonal and the equation obtained for the projected motion along the diagonals is not a Telegrapher equation in our case.

Thus, it is to noted that in these models without angular symmetry, such a description of projected motion is non-unique and depends on the direction of the projected motion. It should, however, be pointed out that the angular spacing between these discrete directions, given by the ratio of the total solid angle to the number of directions, in our model is

$$
\frac{\Omega(d)}{2^{d}}=\frac{1}{2^{d}} \frac{2 \pi^{d / 2}}{\Gamma(d / 2) d} \rightarrow\left(\frac{\pi^{1 / 2} e^{1 / 2}}{2 \ln ^{1 / 2}(d / 2)}\right)^{d},
$$

where $\Gamma$ here is the Gamma function. In the limit of large $d$, the angular spacing decays almost exponentially to zero. Thus, in the limit of large dimensions, this process indeed describes a genuine diffusion at constant speed. This result is due to the exponential de- 
pendence on the dimensionality for the number of allowed directions for the photon velocity and is not obtained by Boguñá et al.

\section{KUBO-ANDERSON LIKE STOCHASTIC PROCESSES}

In passing, we would like to touch upon the possible generalization to more complex stochastic processes, which demonstrates the power of the current approach. It is a simple matter now, to write down the equations for probability distribution function for a KuboAnderson like process, given by the sum of n-independent dichotomic Markov processes (in one-dimension), i.e., $v(t)=v_{1}(t)+v_{2}(t)+\cdots+v_{n}(t)$, where $v_{i}(t)$ can take on the values $\pm c$ and $\left\langle v_{i}(t)\right\rangle=0 ;\left\langle v_{i}(t) v_{j}\left(t^{\prime}\right)\right\rangle=c^{2} \exp \left(-\Gamma\left|t-t^{\prime}\right|\right) \delta_{i j}$. The structure of the equations for $n=2$ and $n=3$ remain the same as Eqn. (16-19) and Eqn. (21-28) respectively, with only the derivatives $\partial / \partial y$ and $\partial / \partial z$ both replaced by $\partial / \partial x$. It is immediately seen that the case of $n=2$ corresponds to the 3 -step Telegrapher processes described above. The generalization to higher $n$ is obvious. In general, one obtains $n+1$ coupled partial differential equations for the sum of $n$-independent dichotomic Markov processes.

\section{CONCLUSIONS}

In conclusion, we have developed a particular 'generalization' of the Telegrapher process to higher dimensional $(d>1)$ stochastic media which could be potentially useful for studying photon migration in turbid media as it rigourously preserves the photon speed to be constant between the scattering events. In comparision to the model presented in Ref. [5], where the photon's random walk was modelled as a diffusion on the velocity sphere, we have a model phase space here, where the photon can move only along the $2^{d}$ directions of the diagonals of the $d$ dimensional hypercube. It is admittedly an artificial phase space, but one having an appreciable directional persistence unlike the zero-persistence diffusion theory. However, the model does not have angular symmetry. In 1-D, there are only two directions and hence, the Telegrapher equation is exact. On the other hand, in the limit of very large dimensions, 
the angular spacing between the directions tends to zero almost exponentially, and again the process is indeed a genuine diffusion-at-a-constant-speed. It has been shown that the equation for the projected motion along any hypercube axis is a 1-D Telegrapher equation, though it is non-invariant under an arbitrary rotation. Further, the ad-hoc generalized Telegrapher equation in higher dimensions [12,[13] is recovered when higher order correlations are neglected. The power of this approach is demonstrated by deriving the equations for a sum of n-independent Markov processes. 


\section{REFERENCES}

[1] S. Chandrasekhar, Radiative transfer, (Dover, 1950).

[2] Akira Ishimaru, Wave Propagation and scattering in random media, Vol.1 \& 2 (Academic, New York, 1978).

[3] K.M. Yoo, F. Liu and R.R. Alfano, Phys. Rev. Lett., 64, 2647, (1990).

[4] V.V. Tuchin and B.J. Thomson, Selected papers on tissue Optics : Applications in medical diagnostics, (SPIE Milestone series, Vol MS 102, Washington, 1990).

[5] S. Anantha Ramakrishna and N. Kumar, Phys. Rev. E 601381 (1999).

[6] S. Goldstein, Quart. J. Math. Appl. Mech. 4, 129 (1951).

[7] J. Maddox, Nature 338, 373 (1989).

[8] J.C. Maxwell, Phil. Trans. Roy Soc. Lond. 157, 49 (1867).

[9] D.D. Joseph and L. Preziosi, Rev. Mod. Phys. 61, 41 (1989).

[10] J.C. Maxwell, in The Collected Papers of J.C. Maxwell, ed. by W.D. Niven (Dover, New York, 1965); and C. Cattaneo, C. R. Acad. Sci 247, 431 (1958).

[11] A. Ishimaru, Appl. Opt. 28, 2210 (1989).

[12] D.J. Durian and J. Rudnick, J. Opt. Soc. Am. A 14, 235 (1997).

[13] D. J. Durian, Opt. Lett. 23, 1502 (1998).

[14] P.A. Lemieux, M.U. Vera and D.J. Durian, Phys. Rev. E, 57, 4498 (1998).

[15] J.M. Porrà, J. Masoliver and G. Weiss, Phys. Rev. E 55, 7771 (1997)

[16] P. M. Morse and H. Feshbach, Methods of Theoretical Physics, vol. I, (McGraw Hill, New York, 1953).

[17] V.E. Shapiro and V.M. Loginov, Physica 91 A, 563 (1978). 
[18] M. Boguñá, J.M. Porrà and J. Masoliver, Phys. Rev. E 58, 6992 (1998).

[19] M. Boguñá, J.M. Porrà and J. Masoliver, Phys. Rev. E 59, 6517 (1999).

[20] N.G. van Kampen, Phys. Rep. 24C, 172, (1976). 